\title{
Síndrome confusional agudo por colirio de cidopentolato
}

\author{
R. J. Romero de CAstilla Gil, E. Fernández Romero*, \\ E. DEL CAMPO Molina** \\ Doctor en Mediana y Cirugía por la U niversidad de Córdoba. Especialista en \\ Medicina Familiar y Comunitaria.*Espedalista en M ediana Familiar y \\ Comunitaria. **FEA de Medicina Intensiva. \\ Hospital Alto Guadalquivir. Andújar. J aén
}

\section{RESUMEN}

El Síndrome Confusional Agudo es un proceso de origen multifactorial; cuando es reversible, las causas más frecuentes son la deprivación de dro gas y los efectos de algunos fármacos, como los agentes parasimpaticolíticos. En oftalmología, es tos fármacos se emplean para provocar midriasis y parálisis de la acomodación en distintas circuns tancias, como tratamiento de queratitis o en el examen del fondo de ojo. Debe recordarse que su uso como gotas o pomadas también puede provocar efectos sistémicos. Muchos de ellos son dependien tes de la dosis, y deben ser conocidos por los profesionales que los emplean.

Presentamos el caso de una mujer joven con un síndrome confusional agudo secundario a adminis tración de ciclopentolato como gotas oculares a dosis habituales. Pensamos que puede ser de inte rés, ya que la mayoría de estos cuadros suelen presentarse en niños y ancianos o asociados a do sis elevadas.

Palabras clave: Ciclopentolato. Síndrome con fusional agudo. Gotas oculares.
Acute confusional state due to cyclopentolate eye drops

\begin{abstract}
The Acute Confusional State is a process with a multifactorial origin; when reversible, the most fre cuents are due to the deprivation of drugs and the effects of some of them, as the parasympatholiticm agents. These drugs are used in ophtalmology to obtein mydriasis and cycloplegic activity in the treatment of several cases as well as keratitis or fundus oculi examination. We can not forget that it use as eye drops or ointment can produce systemic side effects. Many of them appair to be dose-related, and have to be known by the physician who use them.

We report the case of a young women with acute confusional state following the administration of cyclopentolate drops at normal doses. We think it could be interesting, because most of these cases use to happen in children or elderly people or in the case of high doses.
\end{abstract}

Key words: Cyclopentolate. Acute confusional state. Eye drops.

\section{INTRODUCCIÓN}

El progreso en la capacitación de los médicos de familia para el manejo de diversas patologías frecuentes ha propiciado que en su consulta maneje cada vez un número mayor de grupos farmacológicos. Es bien conocido el problema de las interacciones medicamentosas en pacientes polimedicados, ancianos o con patología de base significativa, pero incluso medicamentos ampliamente utilizados

Aceptación: 28-11-01 
en población de riesgo teórico menor pueden tener efectos secundarios poco recordados que posibiliten situaciones comprometidas. Algunos de estos efectos adversos pueden ser leves, pero otros pueden cursar con síntomas llamativos causantes de gran angustia familiar, que no pocas veces derivan en agresividad y posturas demandantes hacia el médico prescriptor. La seguridad en el reconocimiento y manejo de estas reacciones adversas contribuirá a modular estas actitudes no deseables. Uno de los síndromes que, pensamos, más pueden distorsionar la relación terapéutica, sobre todo si aparece en una persona previamente sana y normofuncional, es el síndrome confusional agudo. En la práctica médica diaria existen fármacos de amplia prescripción que pueden causar síntomas y signos de afectación del sistema nervioso central (SNC): alucinaciones, convulsiones, confusión, excitación, coma, síndrome parkinsoniano o cuadros psicóticos. Estas patologías neurológicas y psiquiátricas producidas por fármacos pueden estar relacionadas con la dosis administrada o bien ser reacciones idiosincráticas, impredecibles e independientes de la dosis.

Presentamos un caso de síndrome confusional agudo en una paciente joven y sin antecedentes de interés.

\section{OBSERVACIÓN CLÍNICA}

Se trata de una mujer de 25 años sin ningún antecedente significativo que fue remitida a la consulta del oftalmólogo para graduación por sospecha de defecto de refracción. El día de la consulta se procedió a midriasis y parálisis yatrógena de acomodación con tres gotas en cada ojo de un preparado comercial de ciclopentolato. Aproximadamente tras una hora, ya terminada la consulta del oftalmólogo y antes de marcharse del hospital, es remitida a urgencias por un cuadro súbito de alteración del comportamiento. A su llegada a nuestro servicio, la paciente presenta buen estado general, está bien hidratada y perfundida, hemodinámicamente estable con TA de 140/70, frecuencia cardiaca de 95 lpm y temperatura timpánica de $37,6^{\circ} \mathrm{C}$. La glucemia capilar era de $98 \mathrm{mg} / \mathrm{dl}$. No se aprecia focalidad neurológica motora. La exploración pupilar muestra midriasis arreactiva. Sobresale en la exploración ideación delirante con producción alucinatoria visual de temática diversa (hombres que atraviesan las paredes, animales) de forma intermitente, con discurso coherente entre dichos episodios, en los que nos refiere no notar ningún otro síntoma; todo ello impresiona de brote psicótico. El EKG muestra un ritmo sinusal a $80 \mathrm{lpm}$ sin alteraciones de repolarización. Se extrae analítica y bioquímica general con resultados normales, y su familia nos reitera la inexistencia de antecedentes psiquiátricos o hábitos tóxicos conocidos. Confirmada la administración de ciclopentolato por el servicio de oftalmología, la paciente pasa a observación con diagnóstico de presunción de Síndrome Confusional Agudo secundario a reacción adversa neurológica por anticolinérgicos tópicos conjuntivales. No se juzgó necesario tratamiento antidótico debido a la ausencia de otros síntomas antimuscarínicos y a la evolución favorable. Durante su estancia va remitiendo el cuadro a la vez que la enferma hace crítica del delirio y comenta de forma objetiva sus alucinaciones. Tras unas 8 horas la paciente queda asintomática, permaneciendo así en posteriores controles a las 24 y 48 horas en su Centro de Salud.

\section{DISCUSIÓN}

Se denomina Síndrome Confusional Agudo (SCA) o delirium al cuadro clínico de inicio agudo caracterizado por la aparición fluctuante y concurrente de alteración en el nivel de consciencia y en la atención, así como en todas o alguna de las siguientes áreas: pensamiento, lenguaje, memoria, percepción, comportamiento psicomotor, emoción y ciclo vigilia/sueño. En la literatura se encuentra una rica sinonimia para definir esta situación clínica (encefalopatía metabólica, psicosis tóxica, psicosis externa, síndrome cerebral agudo). Desde el punto de vista fisiopatológico, el SCA se puede producir por alteración funcional cerebral bilateral y difusa, o por lesiones críticas en la sustancia reticular activadora ascendente. También pueden manifestarse como SCA lesiones focales en los lóbulos frontal y parietal derechos o en la circunvolución del cíngulo. Los fármacos o tóxicos y la abstinencia brusca de sustancias como el alcohol (delirium tremens) son las causas más frecuentes de SCA reversible. Entre los medicamentos, los psicofármacos y los anticolinérgicos son los más frecuentemente implicados ${ }^{1}$. En la tabla I se mues-

\section{Tabla I}

FÁRMACOS DE USO FRECUENTE IMPLICADOS EN EL SÍNDROME CONFUSIONAL AGUDO

\begin{tabular}{lcc}
\hline \multicolumn{1}{c}{ AINEs } & Barbitúricos & Levodopa \\
Antiarrítmicos & Benzodiazepinas & Litio \\
Antibióticos & Ciclosporina & Narcóticos \\
Anticomiciales & Cimetidina & Neurolépticos \\
Antidepresivos & Clonidina & Omeprazol \\
Antihipertensivos & Corticoides & Parasimpaticolíticos \\
Antineoplásicos & Digoxina & Sales de bismuto \\
Agonistas & & \\
dopaminérgicos & Interferón & \\
\end{tabular}


tra una relación de fármacos de empleo frecuente que pueden causar síndrome confusional.

A modo de recuerdo fisiológico, las drogas anticolinérgicas se dividen, de acuerdo con el tipo de receptor que bloquean, en dos grandes grupos:

1. Bloqueadores muscarínicos: anticolinérgicos postganglionares o verdaderos parasimpaticolíticos.

2. Bloqueadores nicotínicos: que a su vez pueden clasificarse en dos subgrupos:

-Anticolinérgicos ganglionares o gangliopléjicos.

-Anticolinérgicos neuromusculares o bloqueadores neuromusculares.

Las acciones específicas del pararasimpático son aquéllas que surgen de la estimulación de los receptores muscarínicos ubicados en la terminal postganglionar neuroefectora. Por eso los agentes antimuscarínicos, bloqueantes postganglionares, son los verdaderos agentes parasimpaticolíticos. El prototipo es la atropina, droga antagonista competitiva de la acetilcolina, que es capaz de desencadenar todas las acciones parasimpaticolíticas. No todos los receptores muscarínicos tienen la misma sensibilidad a la atropina; así, en relación a la dosis empleada, aparecen diversos efectos de forma sumativa:

Dosis bajas de atropina inhiben la secreción salival, bronquial y sudorípara, presentando el enfermo piel seca, pudiendo acompañarse de hipertermia y flush (rubor atropínico) en cara y tronco. Sobre el SNC produce ligera estimulación medular y de centros cerebrales superiores.

Dosis moderadas de atropina producen además midriasis, inhibición de la acomodación con visión cercana borrosa, que puede acompañarse de fotofobia e inyección conjuntival, taquicardia y broncodilatación. Sobre el SNC, puede provocar hipomanía, inquietud, desorientación, alucinaciones o delirio por bloqueo de receptores centrales.

Dosis grandes de atropina inhiben el tono y la motilidad gastrointestinal y del árbol urinario. También toxicidad severa en SNC, con depresión respiratoria por parálisis del centro respiratorio y coma $^{2}$.

Como usos terapéuticos comunes, aparte los oftalmológicos que luego mencionaremos, los anticolinérgicos se emplean en diversos procesos: enfermedad de Parkinson por su acción antitremor; cuadros de hipertonía vagal (como en el infarto agudo de miocardio); en intoxicaciones por agentes anticolinesterásicos; como antiespasmódicos gastrointestinales y en EPOC.

Las particularidades del caso que nos ocupa hacen oportuna una reflexión: pocos son los medicamentos de uso exclusivo en oftalmología; la mayoría de ellos son empleados en otros campos de la medicina y sus efectos tóxicos y contraindicacio- nes están bien establecidos. Son sin embargo sus peculiares formas de presentación como colirios y pomadas lo que provoca una falsa sensación de seguridad; esto puede facilitar un uso poco adecuado a pesar de suponer un peligro potencial.

A medida que su uso se ha generalizado, se han descrito reacciones adversas sistémicas, algunas de extrema gravedad, que son perfectamente explicables por la enorme capacidad de absorción de la mucosa conjuntival y la mucosa nasal. La capacidad de absorción de estas mucosas y la rapidez de paso de algunas drogas a la circulación sistémica tras su admimistración por vía tópica hacen equiparable en ocasiones esta vía a la intravenosa ${ }^{2}$.

Los agentes antimuscarínicos producen, instilados en el saco conjuntival, midriasis y parálisis de la acomodación (cicloplejía). Ambas acciones pueden ser necesarias para el tratamiento de iridociclitis, queratitis y coroiditis. La atropina y escopolamina, anticolinérgicos naturales, son agentes muy potentes y de larga duración; sus acciones oculares pueden persistir durante varios días. En cambio, el ciclopentolato y la tropicamida, derivados sintéticos, tienen un efecto mucho más corto, de 6 a 24 horas. Incluso, en soluciones poco concentradas débiles, estos agentes sólo producen midriasis sin afectar la acomodación. El ciclopentolato es ampliamente usado en oftalmología. En la consulta del médico de familia estos fármacos suelen emplearse tras extracción de cuerpos extraños o ante abrasiones corneales significativas, en el estudio del fondo de ojo y ante la sospecha de uveítis para prevención de sinequias. También podemos manejarlos como prescripción continuada desde oftalmología en el tratamiento de hiphemas, queratitis importantes o para estudio de refracción.

Sus efectos secundarios sistémicos han sido descritos tras administración tópica: agitación, delirio, confusión, ataxia, etc. Se trata de una reacción gráficamente denominada "borrachera ciclopléjica". Estas reacciones parecen ser dosis-dependientes y son más comunes en niños, en los que es muy utilizado con fines diagnósticos. También existen referencias de la mayor susceptibilidad de los ancianos $^{3}$. Raramente han sido comunicadas en adultos tras una dosis normal, lo que confiere interés a nuestro caso $^{4-6}$. Desde 1984 a 1991 se notificaron al Sistema Español de Farmacovigilancia 40 reacciones adversas a fármacos ciclopléjicos tópicos, 29 de ellas de tipo sistémico.

El antecedente de exposición al fármaco permite en la mayoría de las ocasiones realizar un diagnóstico de presunción si se tiene en cuenta la posibilidad del cuadro. De todas formas, será obviamente necesaria una historia clínica correcta en la que se recopilen detalles sobre el estado cognitivo previo del paciente, antecedentes de enfermedades sistémicas, trastornos psiquiátricos, uso de otras medi- 
caciones o drogas ilegales, exposición a tóxicos ambientales o profesionales, traumatismo craneal reciente y, en general, cualquier circunstancia o síntoma previo. Como medidas generales prioritarias, al alcance de todo médico, el examen debe incluir la detección selectiva de signos de irritación meníngea, señales de traumatismo, signos de venopunción y la obtención de constantes vitales. Es aconsejable la realización de glucemia capilar y si se dispone de ello, EKG de 12 derivaciones. Se debe canalizar una vía endovenosa periférica antes del traslado del paciente al medio hospitalario para continuar estudio, y asegurar la vía aerea si existe coma. Una vez en nivel hospitalario, se podrá valorar la pertinencia de otras exploraciones complementarias (punción lumbar, pruebas de imagen, EEG), que no fueron consideradas necesarias en nuestro caso.

Como aproximación terapéutica, en caso de intoxicaciones leves o moderadas sólo se debe realizar una estrecha observación, descender la temperatura corporal si hay fiebre -preferiblemente con medidas físicas- y esperar la eliminación metabólica de la droga, hasta 24-48 horas. En casos más graves, con potencial compromiso vital, se aconseja la administración de agentes parasimpáticomiméticos anticolinesterasa. Es de elección la fisostigmina i.v. lenta, en dosis de 0,5 hasta $2 \mathrm{mg}$ que puede repetirse cada 5-10 minutos, porque atravie- sa con mayor facilidad la barrera hematoencefálica, suprimiendo las alucinaciones y revirtiendo el coma si existiera. Su uso debe ser juicioso porque puede producir bradicardia e hipotensión. Las convulsiones y la agitación se tratarán de forma sintomática con diazepan en bolos de 3-5 mg i.v. lento y haloperidol, en dosis de 2 a $10 \mathrm{mg}$ i.v. que pueden repetirse cada media hora, hasta que aparezcan efectos adversos o resolución del cuadro ${ }^{7}$. En caso de contraindicación, puede usarse tiapride (tiaprizal amp. $100 \mathrm{mg}$ ) en dosis de 400-1200 mg/día.

El episodio debe incluirse en la lista de problemas del paciente para prevenir nuevas exposiciones, si bien no hemos encontrado en nuestra revisión bibliográfica estudios que demuestren de forma clara la existencia de una susceptibilidad personal a los efectos secundarios de anticolinérgicos.

\section{CORRESPONDENCIA:}

Rafael J. Romero de Castilla Gil

Hospital Alto Guadalquivir

Avda. Blas Infante, $\mathrm{s} / \mathrm{n}$

23740 Andújar. Jaén

e-mail: rjromero@ephag.es

\section{Bibliografía}

1. Benito J, Tapias E, Barrios A. Síndrome Confusional Agudo Demencias. En: Acedo MS, Barrios A, Díaz R, Orche S, Sanz RM, editores. Manual de diagnóstico y terapéutica Médica. $4^{\mathrm{a}}$ ed. Madrid: MSD 1998. p. 771-9.

2. Weiner N. Atropina, escopolamina y drogas antimuscarínicas afines. En: Goodman A, Gilman A. Las bases farmacológicas de la terapéutica. $6^{\text {a }}$ ed. Madrid: Ed. Panamericana SA, 1985. p. 133-49.

3. Barker DB, Solomon DA. Potential for mental status changes associated with systemic absorption of anticholinergic ophtalmic medications: concerns in the elderly. Ann Pharmacother 1990; $24: 847-50$
4. Wütrich B, Bigler M, Kuhn M, Zimmerli B, Priske S. Systemic anticholinergic side-effects. Allergy 2000; 55: 788-9.

5. Khurana AK, Ahluwalia BK, Rajan C, Vohra AK. Acute psychosis associated with topical cyclopentolate hydrochloride [carta]. Am J Ophthalmol 1988; 105: 91.

6. Wang MK, Tatane JR. Other systemic effects of eye drops. Br Med J 1974; 1: 453-4.

7. Nogué S, Palomar M. Intoxicación medicamentosa. En: Montejo JC, García de Lorenzo A, Ortiz Leyba C, Bonet A. Manual de Medicina Intensiva. $2^{\mathrm{a}}$ ed. Madrid: Ed. Harcourt SA, 2001. p. 492-5. 